\title{
Estenosis aórtica severa de bajo flujo, bajo gradiente con función sistólica preservada, un reto diagnóstico y terapéutico: ¿qué sabemos a día de hoy?
}

\author{
Mar Erdociaín Perales ${ }^{\mathrm{a}, *}$, Pilar Zuazola Martínez ${ }^{\mathrm{a}}$ y Eduardo Cay Diarte ${ }^{\mathrm{b}}$
}

\author{
a Servicio de Cardiologia, Hospital de la Marina Baixa, Villajoyosa (Alicante), España \\ b Servicio de Cardiologia, Hospital Miguel Servet, Zaragoza, España
}

Recibido el 25 de enero de 2016; aceptado el 5 de mayo de 2016

Disponible en Internet el 21 de julio de 2016

\author{
PALABRAS CLAVE \\ Estenosis aórtica \\ severa; \\ Bajo flujo paradójico; \\ Fracción eyección \\ conservada
}

\begin{abstract}
Resumen La estenosis aórtica severa de flujo bajo, gradiente bajo y la fracción de eyección del ventrículo izquierdo conservada es una entidad frecuente en la práctica clínica. Probablemente, represente una fase más avanzada de la enfermedad con una mayor carga soportada o de más larga data y con una fisiopatología restrictiva. Existen características clínicas, hemodinámicas y ecocardiográficas típicas. Se trata de una entidad infradiagnosticada y como consecuencia infratratada con un claro peor pronóstico con tratamiento conservador. Se necesita en muchos casos el apoyo de la «multiimagen» para un adecuado diagnóstico y elección del momento terapéutico. La sustitución valvular en los pacientes con estenosis aórtica severa y flujo bajo paradójico, parecen tener una mayor mortalidad a corto y largo plazo al compararla en los pacientes con flujo normal y similar si lo hacemos frente a la estenosis aórtica de bajo flujo y fracción de eyección del ventrículo izquierdo deprimida. Existe una menor mortalidad con la implantación transcatéter de válvula aórtica comparándolo frente al tratamiento médico en pacientes con estenosis aórtica severa sintomática con flujo bajo, tanto la fracción de eyección baja como el flujo bajo paradójico, considerados inoperables. No parecen existir evidencias actuales para recomendar el abordaje percutáneo frente al recambio valvular aórtico quirúrgico en los pacientes de alto riesgo quirúrgico con estenosis aórtica con flujo bajo paradójico.

(C) 2016 Sociedad Colombiana de Cardiología y Cirugía Cardiovascular. Publicado por Elsevier España, S.L.U. Este es un artículo Open Access bajo la licencia CC BY-NC-ND (http:// creativecommons.org/licenses/by-nc-nd/4.0/).
\end{abstract}

\footnotetext{
* Autor para correspondencia.

Correo electrónico: mar.erdociain@gmail.com (M. Erdociaín Perales).
} 


\section{KEYWORDS}

Severe aortic stenosis;

Paradoxical low flow; Preserved ejection

fraction

\section{Low-flow, low-gradient severe aortic stenosis with preserved systolic function, a diagnostic and therapeutic goal: What do we know today?}

\begin{abstract}
Low-flow, low-gradient severe aortic stenosis with preserved ejection fraction of the left ventricle is a frequent entity of clinical practice. It can probably represent a more advance phase of the illness with a heavier load or time frame and a restrictive pathophysiology. There are typical clinical, hemodynamic and echocardiographic characteristics. It is an underdiagnosed condition, and as such it is undertreated, with a clearly worse prognosis with conservative treatment. In many cases, the help of «multiimaging» is required for an appropriate diagnosis and choosing the therapeutic moment. Valve replacement in patients with paradoxical low-flow, low-gradient severe aortic stenosis seem to show higher mortality in the short and long term when compared to patients with normal flow, and similar mortality if compared to low-flow aortic stenosis with depressed ejection fraction of the left ventricle. There is a lower mortality with the transcatheter aortic valve implantation in comparison with treatment of patients with symptomatic low-flow severe aortic stenosis, both for a low ejection fraction and for the paradoxical low flow, considered inoperable. No current evidence seems to exist to recommend percutaneous approach versus aortic valve replacement in high surgical risk patients with aortic stenosis with paradoxical low flow severe aortic stenosis.

(c) 2016 Sociedad Colombiana de Cardiología y Cirugía Cardiovascular. Published by Elsevier España, S.L.U. This is an open access article under the CC BY-NC-ND license (http:// creativecommons.org/licenses/by-nc-nd/4.0/).
\end{abstract}

\section{Introducción}

La estenosis aórtica (EAo) representa la valvulopatía más frecuente en Europa. Afecta a un 2-7\% de la población mayor de 65 años de edad. Su etiología más frecuente es la degenerativa por lo que su prevalencia aumenta con la edad, llegando a afectar a más del $6 \%$ de la población mayor de 85 años de edad. El envejecimiento de la población hace presagiar un gran incremento de esta valvulopatía. El recambio de la válvula estenótica es el tratamiento definitivo. El momento de la intervención viene determinado por la gravedad de la estenosis y la sintomatología del paciente. Esta decisión nos plantea a diario retos diagnósticos, así como de elección de la terapia y el momento apropiado. Es fundamental para ello conocer las distintas entidades de estenosis aórtica severa así como sus peculiaridades diagnósticas y pronósticas.

Han sido necesarios años de investigación para el reconocimiento de la EAo severa con bajo flujo y bajo gradiente como una entidad establecida y bien comprendida. Hachicha et al. ${ }^{1}$, observaron que aproximadamente el $35 \%$ de los pacientes con el área valvular aórtica (AVA) indexada $<0,6 \mathrm{~cm}^{2} / \mathrm{m}^{2}$ y la fracción de eyección del ventrículo izquierdo ( $\mathrm{FEVI}$ ) preservada, mayor del $50 \%$, presentaban gradientes transvalvulares bajos y que esta situación se acompañaba en la mayoría de los casos con una reducción del gasto cardiaco. Hemos avanzado mucho en el conocimiento y la perspectiva de la EA o severa como enfermedad que no se limita meramente a la válvula desde entonces. En la actualidad queda obsoleta una definición de la EAo severa que solo atienda a los gradientes y/o el área valvular. La definición clásica basada en un $A V A<1,0 \mathrm{~cm}^{2}$ y/o indexada por superficie corpo$\mathrm{ral}<0,6 \mathrm{~cm}^{2} / \mathrm{m}^{2}$ y/o un gradiente medio de $40 \mathrm{mmHg}$ ha sido sustituida por una conjunción de parámetros clínicos, ecocardiográficos que valoran la válvula aórtica y el ventrículo izquierdo (VI) así como hemodinámicos atendiendo al sistema arterial.

\section{Diagnóstico}

\section{El porqué del bajo flujo-bajo gradiente}

La revisión de numerosos estudios ${ }^{1,2}$, nos permiten resumir las características que acompañan a este tipo concreto de EAo severa de bajo flujo, bajo gradiente y FEVI conservada (tabla 1).

Comparándola con la EAo severa de flujo normal y gradientes elevados, los gradientes transvalvulares son marcadamente inferiores, en el $55 \%$ de los casos el gradiente medio se encuentra por debajo de $30 \mathrm{mmHg}$. Son pacientes con un vi con diámetros telediastólicos y volúmenes telediastólicos inferiores y relativamente más engrosados. Además, presentan una reducción del acortamiento radial y longitudinal de la pared y tendencia a menor FEVI aunque se mantienen dentro del rango normal. Todo ello condiciona una fisiopatología que se asemeja a la restrictiva.

Consecuencia de lo anterior, el volumen sistólico (VS) está disminuido, por debajo de $35 \mathrm{ml} / \mathrm{m}^{2}$. Esto constituye el pilar diagnóstico, ya que los gradientes transvalvulares dependen en gran medida de las variaciones del flujo que atraviesa la válvula al ser una función cuadrática de este. Todos estos hallazgos hacen plantearse que quizá se trata de un estadio más avanzado de la enfermedad, el soporte de una carga hemodinámica mayor o de más larga data por parte del ventrículo izquierdo.

\section{El papel de la carga hemodinámica global}

La causa más frecuente de EAo es la degenerativa. Este proceso tiene muchos puntos en común con la arteriosclerosis 
Tabla 1 Comparativa de los datos ecocardiográficos entre las dos entidades de estenosis aórtica severa de bajo flujo - bajo gradiente frente a la estenosis aórtica severa con flujo normal y gradientes elevados

\begin{tabular}{llll}
\hline & FEVI CONSERVADA FLUJO & FEVI CONSERVADA FLUJO & FEVI DISMINUIDA FLUJO \\
& NORMAL GRADIENTE ELEVADO & BAJO GRADIENTE BAJO & BAJO GRADIENTE BAJO \\
\hline AVA $\left(\mathrm{cm}^{2}\right)$ & $<1$ & $<1$ & $<1$ \\
AVA indexada $\left(\mathrm{cm}^{2} / \mathrm{m}^{2}\right)$ & $<0,6$ & $<0,6$ & $<0,6$ \\
Gradiente medio $\left(\mathrm{mmHg}^{2}\right)$ & $>40$ & $<40$ & $<40$ \\
FEVI $(\%)$ & $>50 \%$ & $>50$ & $<50 \%$ \\
VS indexado $\left(\mathrm{ml} / \mathrm{m}^{2}\right)$ & $>35$ & $<35$ & $<35$ \\
VTI TSVI/VTI VAo & $<0,25$ & $<0,25$ & $<0,25$ \\
DTDVI $(\mathrm{mms})$ & $45-55$ & $<47$ & $>50$ \\
Grosor parietal relativo & $>0,43$ & $>0,50$ & $0,35-0,55$ \\
Disfunción diastólica & Leve-moderada & Moderada-severa & Moderada-severa \\
Strain longitudinal global $(\%)$ & $15-20$ & $<15$ & $<15$ \\
Zva (mmHg/ml/m $\left.{ }^{2}\right)$ & $>4,5$ & $>4,5$ & $>4,5$
\end{tabular}

AVA: área valvular aórtica. FEVI: fracción de eyección del ventrículo izquierdo. VS: volumen sistólico. VTI: integral tiempo-velocidad. TSVI: tracto de salida del ventrículo izquierdo. VAo: valvular aórtico. Zva: impedancia válvulo-arterial.

siendo concomitante en muchos de estos pacientes la presencia de hipertensión arterial (HTA) sistólica, una disminución de la distensibilidad arterial sistémica y alteraciones en la función del vı por hipertensión o enfermedad arterial coronaria asociada. La fisiopatología de la EAo es esencialmente un desequilibrio entre el aumento de la carga hemodinámica y la capacidad del vı para superarla. La enfermedad no se limita a la válvula sino que también implica al sistema vascular por lo que hay que tener en cuenta las resistencias arteriales sistémicas y aquí es donde se introduce el concepto de la impedancia válvulo-arterial (Zva).

Este parámetro traduce el costo en $\mathrm{mmHg}$ por cada mililitro de sangre bombeado por el vı indexada por superficie corporal. Su valor normal es menor de $4,5 \mathrm{mmHg} / \mathrm{ml} / \mathrm{m}^{2}$. Es de suma importancia realizar un control de la TA en el momento de realizar el examen ecocardiográfico si queremos hacer una adecuada valoración hemodinámica. El papel de la carga hemodinámica global explica la posible existencia de sintomatología en un paciente con EAo moderada con HTA o alteración de la distensibilidad arterial. A continuación se muestra la fórmula de la impedancia válvulo-arterial según el método descrito por Brian et al. ${ }^{3}$.

$$
\begin{aligned}
\text { Zva }= & \text { gradiente medio VAo } \\
& + \text { TAS / volumen sistólico indexado }
\end{aligned}
$$

\section{Errores frecuentes de medición}

Desde el punto de vista práctico, durante la realización de un examen ecocardiográfico destinado a valorar la severidad de una EAo debemos ser sistemáticos y calcular en todos los pacientes el área valvular por la ecuación de continuidad (EC) así como los gradientes transvalvulares y la FEVI. Debe llamar nuestra atención un AVA $<1 \mathrm{~cm}^{2}$ que se acompaña de gradiente medio transvalvular menor de $40 \mathrm{mmHg}$ y una $\mathrm{FEVI}>50 \%$. En este caso el primer paso a seguir es indexar el AVA por la superficie corporal (SC) ya que si se trata de una persona con SC pequeña tras este simple ajuste podemos objetivar un AVA indexada (AVA i) $>0,60 \mathrm{~cm}^{2}$ y confirmar que todos los datos sugieren una EAo moderada. Si
Tabla 2 Parámetros ecocardiográficos menos dependientes del flujo útiles para el diagnóstico de estenosis aórtica severa

\begin{tabular}{ll}
\hline & EAo severa \\
\hline AVA $\left(\mathrm{cm}^{2}\right)$ & $<1$ \\
AVA indexada $\left(\mathrm{cm}^{2} / \mathrm{m}^{2}\right)$ & $<0,6$ \\
VTI TSVI/VTI VAo & $<0,25$ \\
Resistencia VAo (dinas/s/ $\left.\mathrm{cm}^{5}\right)$ & $>250$ \\
Relación Tiempo de aceleración/Tiempo de & $>0,36$ \\
$\quad$ eyección $(\mathrm{Tac} / \mathrm{TEVI})$ & $>40-50$ \\
Índice de pérdida de trabajo-latido & \\
$\quad$ ventricular $(\%)$ & $>4,5$ \\
Zva $\left(\mathrm{mmHg} / \mathrm{ml} / \mathrm{m}^{2}\right)$ & \\
\hline
\end{tabular}

AVA: área valvular aórtica. VTI: integral tiempo-velocidad. TSVI: tracto de salida del ventrículo izquierdo. VAo: valvular aórtico. Tac: tiempo de aceleración. TEVI: tiempo de eyección del ventrículo izquierdo. Zva: impedancia válvulo-arterial.

no es así, se revisan las medidas donde frecuentemente se producen errores: tracto de salida del VI (TSVI), integral tiempo-velocidad TSVI (VTI TSVI), corroborar el gradiente desde distintos accesos, precisar la FEVI y calcular el VS. En caso de discrepancias debemos completar el examen ecocardiográfico con el cálculo de parámetros poco dependientes del flujo transvalvular que puedan ayudarnos a aclarar el diagnóstico, (tabla 2).

El error potencial en la medición del TSVI, con tendencia a infraestimarlo, se sospecha que es causa de mala clasificación en algunos estudios y así se advierte en las guías de cuantificación de las valvulopatías. El hecho de que la morfología del TSVI no sea circular ha impulsado el empleo de otros métodos de imagen para mejorar la fiabilidad de la medida. González Canovas et al. ${ }^{4}$, calcularon el AVA por planimetría mediante la ecocardiografía transesofágica tridimensional (ETE-3D) a 63 pacientes diagnosticados de EAo severa paradójica por ecocardiografía transtorácica $2 \mathrm{D}$ (ETT- 2D), basasándose en AVA por EC $<1 \mathrm{~cm}^{2}$ y gradiente medio $<40 \mathrm{mmHg}$. Se objetivó una buena concordancia entre ambas técnicas. En el $85,2 \%$ de los casos se obtuvo un 
AVA por planimetría $3 \mathrm{D}<1 \mathrm{~cm}^{2}$. El $14,7 \%$ de ellos tenían un área valvular por planimetría ETE $3 \mathrm{D}>1 \mathrm{~cm}^{2}$. Estos pacientes discordantes presentaban AVA por ETT $2 \mathrm{D}$ ligeramente superiores, pudiéndose tratar de EAo en el límite de la severidad. Asimismo, al utilizar el área del TSVI obtenida por ETE-3D para el cálculo del AVA por EC, nueve pacientes se reclasificaron en un área mayor de $1 \mathrm{~cm}^{2}$. No obstante, el acuerdo entre la planimetría ETE-3D y la evaluación mixta fue pobre $(k=0,28)$. Los resultados anteriores ponen de manifiesto la variabilidad en el cálculo del TSVI y AVA entre técnicas, si bien la planimetría no deja de ser un criterio anatómico no superponible al AVA por EC.

\section{¿Estenosis aórtica severa paradójica o pseudosevera?}

De lo anterior podríamos deducir que al igual que ocurre con los pacientes con EAo bajo flujo, bajo gradiente y FEVI disminuida si aumentamos el flujo transvalvular mediante pruebas de estrés se plantean dos posibilidades: a) Que se trate de una EAo pseudosevera, es decir, una válvula estenótica de grado moderado con apertura incompleta que al aumentar el flujo a su través mejore su apertura disminuyendo en consecuencia los gradientes, y b) Que la EAo sea realmente severa y el área valvular se mantenga en rango de severidad pese al aumento del volumen sistólico (VS).

Esta discriminación es importante porque los pacientes con EAo severa y síntomas, en general, se benefician del recambio valvular aórtico (RVAo) mientras que los pacientes con pseudoestenosis pueden no beneficiarse de la cirugía precisando un estrecho seguimiento ${ }^{5,6}$.

Los cambios en el gradiente y en el AVA durante el estrés dependen en gran medida del aumento del flujo conseguido en el pico del estrés variando considerablemente de un paciente a otro. Clavel et al. ${ }^{7}$, demostraron la aplicabilidad del AVA proyectada durante las pruebas de estrés bien con el ejercicio o con la dobutamina. Para solventar la limitación de la variabilidad del aumento de flujo propusieron el cálculo del AVA indexada proyectada con la ecuación mostrada a continuación:

$$
A V A_{\text {proj }}=\frac{A V A_{\text {peak }}-A V A_{\text {rest }}}{Q_{\text {peak }}-Q_{\text {rest }}} \times\left(250-Q_{\text {rest }}\right)+A V A_{\text {rest }}
$$

El AVA proyectada indexada refleja la hipotética apertura de la válvula si el flujo transvalvular fuera normal calculada según la tendencia durante la prueba, es decir, según el aumento de AVA en $\mathrm{cm}^{2} / \mathrm{m}^{2}$ por cada aumento de flujo (Q) en $\mathrm{ml} / \mathrm{seg}$. Se considera que $250 \mathrm{ml} / \mathrm{seg}$ es el flujo suficiente para obtener una apertura valvular por encima de $0,6 \mathrm{~cm}^{2} / \mathrm{m}^{2}$ si no existe una EAo verdaderamente severa. En este estudio un AVA proyectada indexada menor de $0,55 \mathrm{~cm}^{2} / \mathrm{m}^{2}$ fue el parámetro que mejor predijo la existencia de EAo severa verdadera tras contrastar con el análisis postexplante de la válvula y el riesgo de eventos, muerte o recambio valvular, frente al resto de los parámetros ecocardiográficos habituales. Para que dicha ecuación se pueda aplicar es necesario un aumento del flujo de al menos el $15 \%$. En los casos en los que no se alcance dicho valor, jugaría un importante papel la cuantificación del calcio valvular aórtico (CVA) con la tomografia computarizada multidetector.
La cuantificación del calcio valvular ha demostrado una buena correlación con la severidad hemodinámica de la $E A o^{8-10}$. Esta relación es sexo dependiente. Las mujeres presentan EAo hemodinámicamente severa con menor cantidad de calcio valvular comparado con los hombres incluso después de ajustar por la superficie corporal y por el área seccional del anillo aórtico como consecuencia, los puntos de cortes para identificar EAo severa son más bajos en las mujeres (CVA > $1200 \mathrm{AU}$ ) que en hombres (CVA > 2000). Los valores de corte tras indexar por el área del corte seccional del anillo aórtico, «densidad del CVA», son > $290 \mathrm{AU} / \mathrm{cm}^{2}$ $y>480 \mathrm{UA} / \mathrm{cm}^{2}$, respectivamente ${ }^{10}$, (fig. 1).

La resonancia magnética cardiaca es una importante herramienta diagnóstica y que aporta información pronóstica en esta entidad. Las imágenes de cine permiten planimetrar el AVA anatómica y aportan una medida más exacta del área del TSVI teniendo en cuenta su forma elíptica. Mediante las secuencias de contraste de fase podemos obtener el AVA por EC. La infraestimación del área del TSVI por parte de la ETT es compensada por la sobrestimación del VTI del TSVI por parte de la resonancia magnética cardiaca, resultando en una buena correlación de ambas técnicas ${ }^{11}$.

Por otro lado la existencia de fibrosis, predominantemente subendocárdica, en las secuencias de realce tardío de la resonancia magnética cardiaca, es más frecuente en la EAo severa con bajo flujo que con flujo normal $(100 \%$ vs. $53 \%, p<0,05)$. Este hecho parece relacionarse ecocardiográficamente con una reducción del strain rate longitudinal $(-0,6 \pm 0,2$ vs. $-1,1 \pm 0,3, p<0,05)$ y del desplazamiento del anillo mitral $(5,8 \pm 1,1$ vs. $9,4 \pm 2,4$, $p<0,05)$ en los pacientes con EAo severa de flujo bajo-gradiente bajo ${ }^{13,14}$. Además, la cuantificación de la fibrosis miocárdica parece ser útil en la estratificación del riesgo de los pacientes con EAo severa «clásica» y en los de bajo flujo. Los pacientes con fibrosis miocárdica extensa tienes peores resultados con una terapia conservadora pero a su vez mayor mortalidad quirúrgica y menor regresión de síntomas o mejoría de parámetros ecocardiográficos tras la sustitución valvular aórtica ${ }^{12,13}$.

\section{La anatomía vs. la funcionalidad}

Un adecuado enfoque de la EAo severa de bajo flujo, bajo gradiente y FEVI conservada se basa en la valoración clínica minuciosa apoyada en la «multiimagen» teniendo en cuenta los factores hemodinámicos modificables, como la HTA. No debemos olvidarnos del fenómeno de contracción de flujo que hace insuficiente las técnicas meramente anátomicas (planimetría del AVA). Tenemos que intentar aproximarnos a la funcionalidad mediante el AVA efectiva que es la que condiciona la sobrecarga de presión sobre el vi. Su cálculo a través del AVA por la ecuación de continuidad (EC) es el método más sencillo.

De todas formas, ante la presencia de discordancias, por ejemplo: pacientes con AVA efectiva $<1 \mathrm{~cm}^{2}$, por la ecocardiografía que presenten un AVA anatómica $>1,5 \mathrm{~cm}^{2}$ por tomografía computarizada (TC) o resonancia magnética cardiaca (RMC) debemos plantearnos errores en la medición de AVA por $\mathrm{EC}^{14}$.

Por otro lado, siguen existiendo controversias en cuanto a la correspondencia entre los gradientes transvalvulares y 


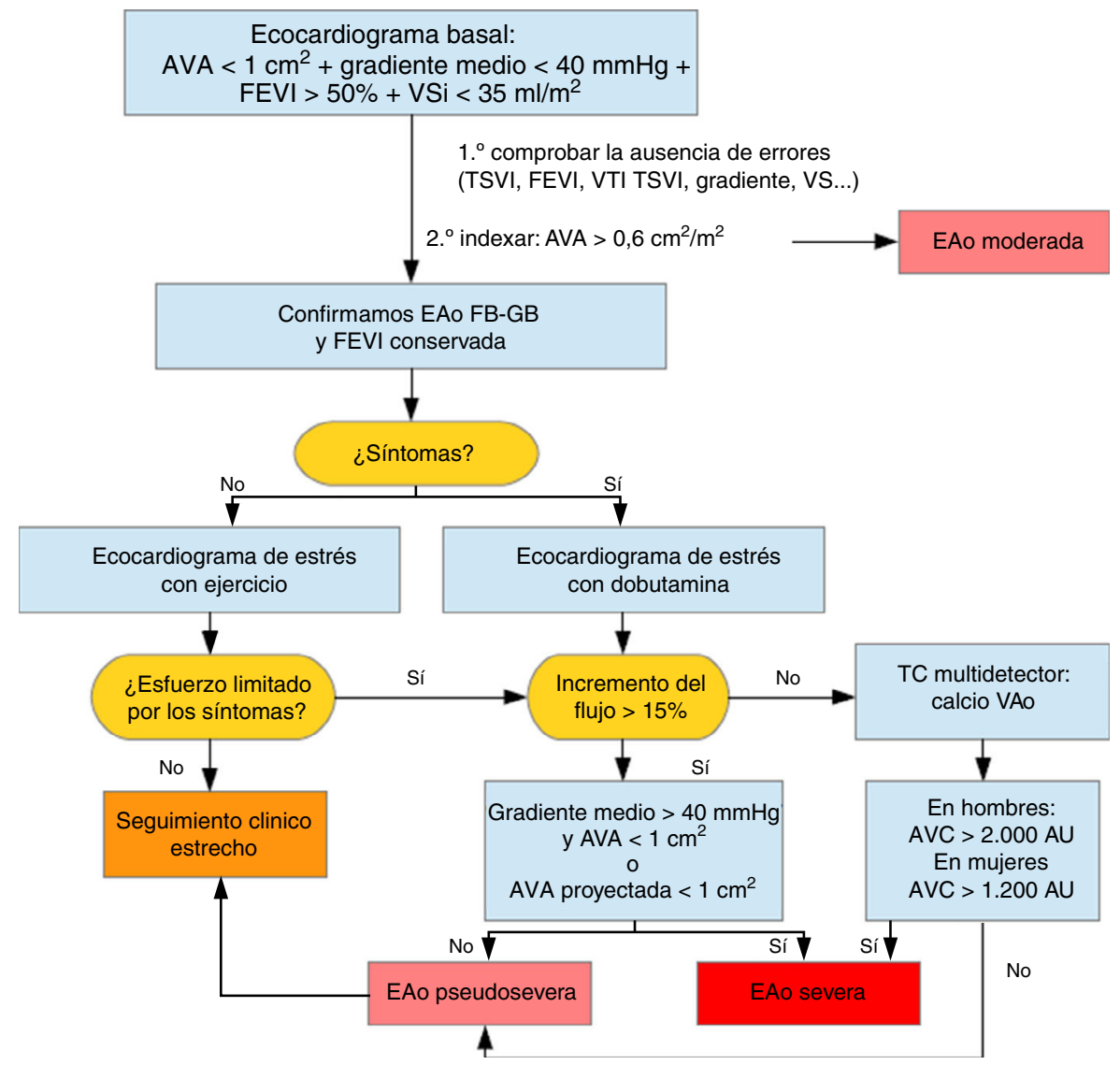

Figura 1 Algoritmo diagnóstico de la EAo severa de flujo bajo, gradiente bajo y FEVI conservada.

una determinada AVA. Podemos clasificar a los pacientes con un AVA indexada $<0,6 \mathrm{~cm}^{2} / \mathrm{m}^{2}$ y $\mathrm{FEVI}$ conservada en cuatro grupos atendiendo al gradiente medio y al volumen sistólico (VS) en: flujo normal (FN) con gradiente alto (GA), FN con gradiente bajo (GB), flujo bajo (FB) con $G A$ y $F B$ con $\mathrm{GB}^{15}$. El grupo de FN con GB plantea varias hipótesis: la existencia de fallos en las medidas, una mala indexación en los pacientes con una superficie corporal pequeña que sobrestima la severidad de la estenosis o quizá replantea el hecho de que un área de $1 \mathrm{~cm}^{2}$ genera un gradiente medio menor. Por ejemplo: en base a la fórmula de Gorlin y suponiendo un gasto cardiaco normal, un AVA de $1,0 \mathrm{~cm}^{2}$ produce un gradiente medio de $26 \mathrm{mmHg}$ y un AVA menor a $0,81 \mathrm{~cm}^{2}$ es necesaria para producir un gradiente mayor de $40 \mathrm{mmHg}^{16-18}$. El pronóstico de este grupo, FN-GB, al igual que los otros tres grupos, es significativamente peor si no se someten a tratamiento quirúrgico ${ }^{10}$.

\section{Tratamiento}

\section{El infradiagnóstico y el consecuente infratratamiento}

El primer factor que condiciona el pronóstico de los pacientes con EAo severa de FB-GB es el infradiagnóstico y el consecuente infratratamiento. Clavel et al. ${ }^{19}$, compararon a 187 pacientes con EAo severa de FB-GB y FEVI conservada con el mismo número de pacientes, por un lado atendiendo al área valvular, $\mathrm{AVA}<0,6 \mathrm{~cm}^{2} / \mathrm{m}^{2}$, grupo de EAo severa de
FN- GA y por otro basándose en los gradientes, con un gradiente medio menos de $40 \mathrm{mmHg}$ y $A V A>0,6 \mathrm{~cm}^{2} / \mathrm{m}^{2}$, es decir, con EAo moderada. Durante un seguimiento medio de 4,2 años, los pacientes con EAo severa FB-GB tuvieron una incidencia más baja de RVAo ( $55 \%$ a 5 años) comparado con el grupo de FN- GA (85\% a 5 años) y más alta si se comparaba con el grupo de EAo moderada (47\% a 5 años). Los pacientes con FB-GB presentaron una supervivencia global y cardiovascular menor comparada con los otros dos grupos, FN-GA y EAo moderada (global: $64 \%$ a 5 años, 82 y $81 \%$ y CV: 74, 85 y 91\%, respectivamente). El RVAo se asoció a una mejor supervivencia en la cohorte completa (HR: 0,35; 95\% $\mathrm{Cl}: 0,21$ a 0,56, $\mathrm{p}<0,0001$ ), así como en los grupos de EAo FN-GA (HR: 0,18; 95\% Cl: 0,07 a 0,48, $\mathrm{p}=0,001$ ) y $\mathrm{FB}-\mathrm{GB}$ (HR: 0,50; 95\% Cl: 0,25 a 0,99, $\mathrm{p}=0,04$ ) con una tendencia no significativa en el grupo EAo moderada.

\section{Nivel de evidencia actual}

Diversos estudios han demostrado que los pacientes con EAo severa FB-GF y FEVI conservada sintomática se benefician del RVAo ${ }^{15,19,23}$, y así ha quedado reflejado recientemente, en las directrices de nuestra práctica clínica. En las Guías Europeas de Valvulopatías del año $2012^{20}$, apuntan que, en pacientes sintomáticos con EAo con flujos y gradientes bajos (< $40 \mathrm{mmHg}$ ) y FEVI normal, solo se debe considerar la RVAo tras una evaluación meticulosa y confirmación de la gravedad de la EAo, con una grado de recomendación lla nivel de evidencia B. En la guías de la American College of 
Cardiology/American Heart Association ${ }^{14}$, y con el mismo nivel de evidencia afirman que el RVAo es razonable en pacientes sintomáticos que tienen EAo de $\mathrm{FB} / \mathrm{GB}$ (estadio D3) que están normotensos y tienen una $\mathrm{FEVI}>50 \%$, si los datos clínicos, hemodinámicos y anatómicos apoyan la obstrucción valvular como la causa más probable de los síntomas.

\section{Resultados según el tipo de tratamiento}

Clavel et al. ${ }^{21}$, compararon los resultados del RVAo en pacientes con EAo severa FB-GB y FEVI deprimida (FEB), EAo paradójica (FBP) y EAo severa con flujo normal (FN). La mortalidad a 30 días fue más alta en los grupos FBP y FEB $(6,3 \%$ y $6,3 \%$ vs. $1,8 \%$, respectivamente, $\mathrm{p}<0,0001)$. El VS indexado y FBP fueron predictores independientes de la mortalidad operatoria (odds ratio [OR]: 1,18, $\mathrm{p}<0,05$; y OR: $2,97, p=0,004$; respectivamente). Esto sugiere que el VS debería integrarse en los scores de riesgo operatorio y no atender únicamente a la baja FEVI. Tras 5 años del RVAo, el grupo FEB presentó los peores resultados de supervivencia, mientras que los grupos FBP y FN tuvieron resultados similares tras ajustar por: la edad, el sexo femenino, la clase NYHA III o Iv; la existencia de: la fibrilación auricular, la enfermedad renal crónica, la enfermedad pulmonar obstructiva crónica, la masa vı indexada y el gradiente medio ( $72 \pm 4 \%$ en el grupo FEB, $81 \pm 2 \%$ en el grupo FBP y $85 \pm 2 \%$ en el grupo de FN $(p<0,0001))$. La baja FEVI resultó predictor independiente de la mortalidad a largo plazo no siéndolo el bajo VS.

En el estudio Partner ${ }^{22}$, el $55 \%$ de los pacientes presentaban: FB (VSi $<35 \mathrm{ml} / \mathrm{m}^{2}$ ), 31\% FEVI conservada (FBP) y $23 \%$ FEB. En el análisis por intención de tratar, independientemente de la terapia, el grupo FB presentó mayor mortalidad a dos años comparado con aquellos con un VSi normal $(47 \%$ vs. 34\%; hazard ratio, 1,5; 95\% IC, 1,25-1,89; $p=0,006)$. No existieron diferencias estadísticamente significativas en la mortalidad entre FEB y FBP $(48,7 \%$ vs. $46 \%$, HR: 0,95 ; 95\% Cl: $0,73,1,23 ; p=0,7)$. En el grupo $F B$, atendiendo a la cohorte inoperable, la mortalidad con tratamiento médico fue de un 73\% y con RVAo transcatéter (TAVI) de un $43 \%$ (RR: 0,$49 ; 95 \% \mathrm{Cl}: 0,33-0,72 ; \mathrm{p}=0,0002$ ), mientras que no hubo diferencias significativas entre TAVI y RVAo en la cohorte de alto riesgo, con una mortalidad a 2 años del 39,6 y $38 \%$, respectivamente (RR: 0,86 ; $95 \% \mathrm{Cl}$ : 0,58-1,29; $\mathrm{p}=0,47)$. Si nos centramos específicamente en el grupo de FBP, para los pacientes inoperables, la mortalidad a dos años se redujo de un $73 \%$ con tratamiento médico a un $43 \%$ con TAVI (HR: 0,48; 95\% Cl: 0,28-0,80; $\mathrm{p}=0,004$ ) y no hubo diferencias en los pacientes de alto riesgo entre TAVI y RVAo (mortalidad 39,0\% vs. 38,3\%; HR: 0,91; 95\% Cl: 0,57-1,45; $\mathrm{p}=0,69)$. En el grupo de $F B$, cohorte alto riesgo, después de los 30 primeros días postprocedimiento, las curvas de mortalidad son divergentes con una menor mortalidad a los 6 meses a favor de TAVI comparado con RVAo $(15,6 \%$ vs. 24,7\%; RR: 0,60; 95\% Cl: 0,37-0,98; $p=0,04)$, no obstante, esta diferencia no es significativa al año volviéndose a unir las curvas. En el grupo FBP esta divergencia se prolonga más en el tiempo, las curvas son divergentes hasta más de 400 días postprocedimento, para equipararse posteriormente. $\mathrm{El}$ hallazgo de una mejor supervivencia temprana con TAVI es intrigante. Pueden existir diferentes explicaciones como el carácter menos invasivo del implante percutáneo, el efecto deletéreo de la circulación extracorpórea en pacientes con un flujo y/o reserva contráctil limitada o quizá un mayor orificio efectivo de la válvula percutánea SAPIENS comparada con las bioprótesis standard ${ }^{23}$. En cualquier caso este beneficio inicial no parece traducirse en un beneficio a más largo plazo en este grupo de alto riesgo. Interesantes serán los resultados de los estudios en pacientes de menor riesgo que están por venir.

Florent et al. ${ }^{24}$, analizaron el impacto del bajo flujo en los pacientes de alto riesgo sometidos a TAVI. 334 pacientes $(52,3 \%)$ de los sometidos a TAVI en su estudio presentaban bajo flujo. Se objetivó una mayor mortalidad a 30 días $(11,4$ vs. $5,9 \%, p=0,01)$ y por todas las causas a 2 años $(35,3$ vs. $30,9 \%, p=0,005$ ) comparados con los pacientes con flujo normal. No hubo diferencias en cuanto a mortalidad del grupo de EAo severa paradójica frente al de bajo flujo por FEVI deprimida. Cabe destacar que el grupo con bajo flujo pero altos gradientes presentó resultados de mortalidad similares a los del grupo con flujo normal. Este hecho hace plantear la peculiaridad de esta entidad que parece traducir una mayor severidad de la estenosis pero mejores resultados postoperatorios en probable relación con una mayor reserva contráctil.

\section{Conclusiones}

La estenosis aórtica severa de FB-GB y FEVI conservada es una entidad frecuente en la práctica clínica. Probablemente, represente una fase más avanzada de la enfermedad con una mayor carga soportada o de más larga data y con una fisiopatología restrictiva. Existen características clínicas, hemodinámicas y ecocardiográficas típicas. Se trata de una entidad infradiagnosticada y como consecuencia infratratada con un claro peor pronóstico con el tratamiento conservador. Se necesita en muchos casos el apoyo de la «multiimagen» para un adecuado diagnóstico y elección del momento terapeútico. Hay que destacar el papel principal del VS indexado reducido en el diagnóstico, así como su papel como predictor de mortalidad y el interés de incluirlo en los scores de riesgo preoperatorio. La sustitución valvular en los pacientes con EAo severa FBP parece tener una mayor mortalidad a corto y largo plazo al compararla con los pacientes con flujo normal y similar si lo hacemos frente a la EAo de bajo flujo y FEVI deprimida (pese a los resultados no coincidentes de algunos estudios). Existe una menor mortalidad a dos años con el implante de TAVI comparándolo frente a tratamiento médico en pacientes con EAo severa sintomática con FB, tanto FEB como FBP, considerados inoperables. Si comparamos el abordaje percutáneo y quirúrgico en pacientes de alto riesgo quirúrgico con EAo FBP existe una menor mortalidad inicial con TAVI que se equipara a la quirúrgica una vez superado ligeramente el primer año. Por tanto, no existen evidencias actuales para recomendar el abordaje percutáneo frente al RVAo quirúrgico en este grupo.

\section{Responsabilidades éticas}

Protección de personas y animales. Los autores declaran que para esta investigación no se han realizado experimentos en seres humanos ni en animales. 
Confidencialidad de los datos. Los autores declaran que en este artículo no aparecen datos de pacientes.

Derecho a la privacidad y consentimiento informado. Los autores declaran que en este artículo no aparecen datos de pacientes.

\section{Conflicto de intereses}

Los autores declaran no tener ningún conflicto de intereses.

\section{Agradecimientos}

A los compañeros cardiólogos del Hospital de La Marina Baixa y del Hospital Miguel Servet.

\section{Bibliografía}

1. Hachicha Z, Dumesnil JG, Bogaty P, Pibarot P. Paradoxical low flow, low gradient severe aortic stenosis despite preserved ejection fraction is associated with higher afterload and reduced survival. Circulation. 2007;115:2856-64.

2. Dumesnil JG, Pibarot P, Carabello B. Paradoxical low flow and/or low gradient severe aortic stenosis despite preserved left ventricular ejection fraction: implications for diagnosis and treatment. European Heart Journal. 2010;31:281-9.

3. Briand M, Dumesnil JG, Kadem L, Tongue AG, Rieu R, Garcia D, et al. Reduced systemic arterial compliance impacts significantly on left ventricular afterload and function in aortic stenosis: implications for diagnosis and treatment. J Am Coll Cardiol. 2005;46:291-8.

4. Gonzalez-Canovas C, Muñoz-Esparza C, Oliva MJ, GonzálezCarrillo J, López-Cuenca A, Saura D, et al. Estenosis aórtica grave con bajo gradiente y fracción de eyección normal: ¿una cuestión de mala clasificación? Rev Esp Cardiol. 2013;66:255-60.

5. Fougères É, Tribouilloy $C$, Monchi M, Petit-Eisenmann H, Baleynaud S, Pasquet A, Chauvel C, Metz D, Adams C, Rusinaru D, Guéret $\mathrm{P}$, Monin JL. Outcomes of pseudo-severe aortic stenosis under conservative treatment. Eur Heart J. 2012;33:2426-33.

6. Clavel MA, Messika-Zeitoun D, Pibarot P, Aggarwal SR, Malouf J, Araoz PA, Michelena HI, Cueff C, Larose E, Capoulade R, Vahanian A, Enriquez-Sarano M. The complex nature of discordant severe calcified aortic valve disease grading: new insights from combined Doppler echocardiographic and computed tomographic study. J Am Coll Cardiol. 2013;62:2329-38.

7. Clavel MA, Ennezat PV, Maréchaux S, Dumesnil JG, Capoulade R, Hachicha Z, Mathieu P, Bellouin A, Bergeron S, Meimoun P, Arsenault M, Le Tourneau T, Pasquet A, Couture C, Pibarot P. Stress echocardiography to assess stenosis severity and predict outcome in patients with paradoxical low-flow, low-gradient aortic stenosis and preserved LVEF. JACC Cardiovasc Imaging. 2013;6:175-83.

8. Messika-Zeitoun D, Aubry MC, Detaint D, Bielak LF, Peyser PA, Sheedy PF, Turner ST, Breen JF, Scott C, Tajik AJ, Enriquez-Sarano M. Evaluation and clinical implications of aortic valve calcification measured by electron-beam computed tomography. Circulation. 2004;110:356-62.

9. Cueff C, Serfaty JM, Cimadevilla C, Laissy JP, Himbert D, Tubach F, Duval X, lung B, Enriquez-Sarano M, Vahanian A, MessikaZeitoun D. Measurement of aortic valve calcification using multislice computed tomography: correlation with haemodynamic severity of aortic stenosis and clinical implication for patients with low ejection fraction. Heart. 2011;97:721-6.
10. Aggarwal SR, Clavel MA, Messika-Zeitoun D, Cueff C, Malouf J, Araoz PA, Mankad R, Michelena H, Vahanian A, EnriquezSarano M. Sex differences in aortic valve calcification measured by multidetector computed tomography in aortic stenosis. Circ Cardiovasc Imaging. 2013;6:40-7.

11. García J, Kadem L, Larose E, Clavel MA, Pibarot P. Comparison between cardiovascular magnetic resonance and transthoracic Doppler echocardiography for the estimation of effective orifice area in aortic stenosis. J Cardiovasc Magn Reson. 2011;13:25.

12. Weidemann F, Herrmann S, Störk S, Niemann M, Frantz $S$, Lange V, Beer M, Gattenlöhner S, Voelker W, Ertl G, Strotmann JM. Impact of myocardial fibrosis in patients with symptomatic severe aortic stenosis. Circulation. 2009;120: 577-84.

13. Herrmann S, Stork S, Niemann M, Lange V, Strotmann JM, Frantz S, Beer M, Gattenlöhner S, Voelker W, Ertl G, Weidemann F. Low-gradient aortic valve stenosis myocardial fibrosis and its influence on function and outcome. J Am Coll Cardiol. 2011;58:402-12.

14. Nishimura RA, Otto CM, Bonow RO, Carabello BA, Erwin JP 3rd, Guyton RA, O'Gara PT, Ruiz CE, Skubas NJ, Sorajja P, Sundt TM 3rd, Thomas JD, American College of Cardiology/American Heart Association Task Force on Practice Guidelines. 2014 AHA/ACC guideline for the management of patients with val vular heart disease: executive summary: a report of the American College of Cardiology/American Heart Association Task Force on Practice Guidelines. J Am Coll Cardiol. 2014;63:2438-88.

15. Dumesnil1 JG, Pibarot P, Carabello B. Paradoxical low flow and/or low gradient severe aortic stenosis despite preserved left ventricular ejection fraction: implications for diagnosis and treatment. Eur Heart J. 2010 Feb;31(3):281-9.

16. Minners J, Allgeier M, Gohlke-Baerwolf C, Kienzle RP, Neumann FJ, Jander N. Inconsistencies of echocardiographic criteria for the grading of aortic valve stenosis. Eur Heart J. 2008;29:1043-8.

17. Carabello BA. Aortic stenosis. N Engl J Med. 2002;346:677-82.

18. Jander N. Low-gradient 'severe' aortic stenosis with preserved ejection fraction: new entity, or discrepant definitions? Eur Heart J. 2008;10. E-11-E-15.

19. Clavel MA, Dumesnil JG, Capoulade R, Mathieu P, Sénéchal M, Pibarot P. Outcome of patients with aortic stenosis, small valve area, and low-flow, low-gradient despite preserved left ventricular ejection fraction. J Am Coll Cardiol. 2012;60(14):1259-67.

20. Joint Task Force on the Management of Valvular Heart Disease of the European Society of Cardiology (ESC); European Association for Cardio-Thoracic Surgery (EACTS)Vahanian A, Alfieri $O$, Andreotti F, Antunes MJ, Barón- Esquivias G, Baumgartner $\mathrm{H}$, Borger MA, Carrel TP, De Bonis M, Evangelista A, Falk V, lung B, Lancellotti P, Pierard L, Price S, Schäfers HJ, Schuler G, Stepinska J, Swedberg K, Takkenberg J, Von Oppell UO, Windecker S, Zamorano JL, Zembala M. Guidelines on the management of valvular heart disease (version 2012). Eur Heart J. 2012;33:2451-96.

21. Clavel MA, DVM, Berthelot-Richer M, Le Ven F, Capoulade R, Dahou A, Dumesnil JG, Mathieu P, Pibarot P. Impact of Classic and Paradoxical Low Flow on Survival After Aortic Valve Replacement for Severe Aortic Stenosis. J Am Coll Cardiol. 2015; 65(7):645-653.

22. Howard C. Herrmann, MD; Philippe Pibarot, PhD; Irene Hueter, PhD; Zachary M. Gertz, MD; William J. Stewart, MD; Samir Kapadia, MD; E. Murat Tuzcu, MD; Vasilis Babaliaros, MD; Vinod Thourani, MD; Wilson Y. Szeto, MD; Joseph E. Bavaria, MD; Susheel Kodali, MD; Rebecca T. Hahn, MD; Mathew Williams, MD; D. Craig Miller, MD; Pamela S. Douglas, MD; Martin B. Leon, MD. Predictors of Mortality and Outcomes of Therapy in Low-Flow Severe Aortic Stenosis A Placement of Aortic Transcatheter Valves (PARTNER) Trial Analysis Circulation. 2013; 127:2316-2326. 
23. Clavel MA, Webb JG, Rodés-Cabau J, Masson JB, Dumont E, De Larochellière R, Doyle $D$, Bergeron $\mathrm{S}$, Baumgartner $\mathrm{H}$, Burwash IG, Dumesnil JG, Mundigler G, Moss R, Kempny A, Bagur R, Bergler-Klein J, Gurvitch R, Mathieu P, Pibarot P. Comparison between transcatheter and surgical prosthetic valve implantation in patients with severe aortic stenosis and reduced left ventricular ejection fraction. Circulation. 2010;122:1928-36.
24. Le Ven F, Freeman M, Webb J, Clavel MA, Wheeler M, Éric Dumont E, Thompson C, De Larochellière R, Moss R, Doyle D, Ribeiro HB, Urena M, Nombela-Franco L, Rodés-Cabau J, Pibarot P. Impact of low flow on the outcome of high-risk patients undergoing transcatheter aortic valve replacement. J Am Coll Cardiol. 2013;62:782-9. 